\title{
Theranosties
}

Editorial

2013; 3(4):289-291. doi: 10.7150/thno.6382

\section{Theranostic Upconversion Nanoparticles (I)}

\author{
Guanying Chen ${ }^{1 凶}$ and Gang $\operatorname{Han}^{2} \bowtie$ \\ 1. Institute for Lasers, Photonics, and Biophotonics, University at Buffalo, State University of New York, Buffalo, New York 14260 (USA) \\ 2. Department of Biochemistry and Molecular Pharmacology, University of Massachusetts Medical School, Worcester, Massachusetts \\ 01605 (USA)
}

$\square$ Corresponding author: guanying@buffalo.edu (Guanying Chen) and Gang.Han@umassmed.edu (Gang Han).

(c) Ivyspring International Publisher. This is an open-access article distributed under the terms of the Creative Commons License (http://creativecommons.org/ licenses/by-nc-nd/3.0/). Reproduction is permitted for personal, noncommercial use, provided that the article is in whole, unmodified, and properly cited.

Received: 2013.04.0I; Accepted: 2013.04.01; Published: 2013.04.04

\begin{abstract}
This theme issue provides a comprehensive collection of original research articles on the creation of diverse types of theranostic upconversion nanoparticles, their fundamental interactions in biology, as well as their biophotonic applications in noninvasive diagnostics and therapy.
\end{abstract}

Key words: Upconversion, Bioimaging, Nontoxicity, Plasmonics, Multimodal.

Photon upconversion is a non-linear anti-Stokes process that converts two or more low-energy excitation photons, which are generally near infrared (NIR) light, into higher energy shorter wavelength emissions (e.g., NIR, visible, ultraviolet) through the use of long lifetime and real ladder-like energy levels of trivalent lanthanide ions embedded in an appropriate inorganic host lattice [1-4]. The recent emerging upconversion nanoparticle has created numerous opportunities for diagnostics and therapy due to the integration of the advantages of its unique dimensions as well as its special unnatural optical properties. These nanoparticles have a multitude of uses toward diagnosis and therapy. For example, (1) the NIR emission can allow for high contrast deep tissue imaging, thus ideal for image-guided surgery through which the tumor located deep inside a body can be seen and treated simultaneously, (2) in biology, ultraviolet emissions are capable of activating photochemical reactions. In addition, their unique optical and chemical properties, such as non-blinking, non-photobleaching, absence of autofluorescence, low-toxicity, and low photodamage to the living system further strengthens their use in medical diagnostics and imaging at both single molecule and ensemble levels. Moreover, a new direction for theranostic upconversion nanoparticles is that of building hierarchical nanostructures for various imaging and therapy applications, such as plasmonics, magnetic resonance imaging (MRI), $x$-ray computed tomography (CT) and positron emission tomography (PET) for in vitro and in vivo theranostics.

This issue (volume 3, number 4) and the next issue (volume 3, number 5) of Theranostics, dedicated to "Theranostic Upconversion Nanoparticles", are aimed at covering aspects of forefront development of upconversion nanoparticles in theranostics. The research articles in this issue include nanoparticle toxicity, plasmonic interactions, protein and stem-cell imaging, as well as therapy. Wolfbeis et al. developed protein-reactive multicolor upconverison nanoparticles using a poly(ethylene glycol) spacer and $\mathrm{N}$-hydroxysuccinimide ester groups [5]. Han et al. produced polyethylenimine (PEI) covalently conjugated core/shell $\left(\mathrm{a}-\mathrm{NaYbF}_{4}: \mathrm{Tm}^{3+}\right) / \mathrm{CaF}_{2}$ upconversion nanoparticles for the labeling of rat mesenchymal stem cells (rMSCs). Upon in vitro induction, this exhibited normal osteogenic and adipogenic differentiation [6]. Cui et al. implemented a toxicity assessment of water-dispersed $\mathrm{LaF}_{3}: \mathrm{Yb}, \mathrm{Er}$ upconversion nanoparticles in zebrafish [7]. This study offers a better understanding for the future study of the toxicological 
mechanism of these nanoparticles. A core/shell $\mathrm{NaYF}_{4}: \mathrm{Er}^{3+}, \mathrm{Yb}^{3+} @ \mathrm{SiO}_{2}$ design by Hilderbrand et al. with highly absorbing NIR carbocyanine dyes in the outer silica shell produced a new tool in terms of combining molecular imaging and photo-thermal therapy [8]. Lastly, the interaction of upconversion with plasmonics has been investigated by Lu et al. [9] and Prasad et al. [10] and has displayed impressive plasmonic enhancement of upconversion emissions, as well as optical multimodal imaging capability. All told, this special issue reports on the frontline findings with respect to the theranostic applications of upconversion nanoparticles, which in turn encourages new directions for combined diagnosis and therapy.

Despite recent exciting results in developing theranostic upconversion nanoparticles, challenges remain for those working in the field. In this regard, we ask the following two questions: What is the major hurdle in the development of upconversion nanoparticles for biomedical applications? What may be the next big thing in this area? We surveyed some of the leading scientists in the field and the answers vary.

So, what is the major hurdle in the development of upconversion nanoparticles for biomedical applications? Professors Chunhua Yan and Ling-Dong Sun from Peking University, as well as our theme contributor Professor Otto S. Wolfbeis from the University of Regensburg, believe that it is the production of upconversion nanoparticles in a highly reproducible way. The reproducibility includes size, size distribution, having a consistent crystal structure, spectral properties and surface modifications. While Professor J. A. Capobianco from Concordia University believes that a detailed understanding of the surface chemistry of nanoparticles is the biggest hurdle. In other words, what is missing here is knowledge is the precise interactions between the surface atoms and functional ligands, as well as the interaction between the resulting surface tailored nanoparticles and molecules (e.g., DNA, proteins) in biological cues. In addition, Professor Wenbo $\mathrm{Bu}$ from the Shanghai Institute of Ceramics, the Chinese Academy of Sciences, believes that the largest hurdle in the development of UCNPs for biomedical applications is the active/passive targeting in diagnosis and treatment. On the other hand, Prof. Yong Zhang from the National University of Singapore feels that the biggest hurdle is that the efficiency and emission color cannot satisfy practical applications.

What may be the next big thing in this area? Professors Chunhua Yan and Ling-Dong Sun believe that a shift in excitation wavelength from $980 \mathrm{~nm}$ to a more appropriate wavelength that produces a much lower heating effect is the next big step in the field. Professor
Wenbo $\mathrm{Bu}$ believes that the next big thing is the optimization of particle size, surface characteristics, as well as targeting efficiency in vivo. Professor J. A. Capobianco feels that we must carry out more studies on animals or develop an appropriate model. In addition, new upconverting materials must be synthesized to the point that they show more efficient upconversion. Professor Yong Zhang believes that higher efficiency and more emission colors must be created utilizing lanthanide doping and new host materials or new structure.

To summarize, this is a quite diverse collection of reviews and research articles that offer us an overview of the study of various upconversion nanoparticles for diagnostic and therapeutic applications. We sincerely hope that the intriguing thoughts and opinions expressed by the contributors to this special issue will provide much of the common ground needed to advance the field of theranostic upconversion nanoparticles.

\section{References}

1. Auzel F. Upconversion and anti-Stokes processes with $\mathrm{f}$ and $\mathrm{d}$ ions in solids. Chem Rev. 2004; 104: 139-74.

2. Haase M, Schäfer H. Upconverting Nanoparticles. Angew Chem Int Ed. 2011; 50: 5808-29.

3. Chen G, Yang C, Prasad PN. Nanophotonics and Nanochemistry: Con-trolling the Excitation Dynamics for Frequency Up- and Down-Conversion in Lanthanide-Doped Nanoparticles. Acc Chem Res. 2013; DOI:10.1021/ar300270y.

4. Zhou J, Liu Z, Li F. Upconversion Nanophosphors for Small-Animal Imaging. Chem Soc Rev, 2012, 41: 1323-1349.

5. Wilhelm S, Hirsch T, Patterson WM, Scheucher E, Mayr T, Wolfbeis OS. Multicolor Upconversion Nanoparticles for Protein Conjugation. Theranostics 2013; 3(4):239-248.

6. Zhao L, Kutikov A, Shen J, Duan C, Song J, Han G. Stem Cell Labeling using Polyethylenimine Conjugated $\left(\mathrm{a}-\mathrm{NaYbF}_{4}: \mathrm{Tm}^{3+}\right) / \mathrm{CaF}_{2}$ Upconversion Nanoparticles. Theranostics 2013; 3(4):249-257.

7. Wang K, Ma J, He M, Gao G, Xu H, Sang J, Wang Y, Zhao B, Cui D. Toxicity Assessments of Near-infrared Upconversion Luminescent $\mathrm{LaF}_{3}: \mathrm{Yb}$,Er in Early Development of Zebrafish Embryos. Theranostics 2013; 3(4):258-266.

8. Shan G, Weissleder R, Hilderbrand SA. Upconverting Organic Dye Doped Core-Shell Nano-Composites for Dual-Modality NIR Imaging and Photo-Thermal Therapy. Theranostics 2013;3(4):267-274.

9. Liu S, Chen G, Ohulchanskyy TY, Swihart MT, Prasad PN. Facile Synthesis and Potential Bioimaging Applications of Hybrid Upconverting and Plasmonic $\mathrm{NaGdF}_{4}: \mathrm{Yb}^{3+}, \mathrm{Er}^{3+} /$ Silica/Gold Nanoparticles. Theranostics 2013; 3(4):275-281.

10. Ge W, Zhang XR, Liu M, Lei ZW, Knize RJ, Lu Y. Distance Dependence of Gold-Enhanced Upconversion luminescence in $\mathrm{Au} / \mathrm{SiO} 2 / \mathrm{Y}_{2} \mathrm{O}_{3}: \mathrm{Yb}^{3+}$, $\mathrm{Er}^{3+}$ Nanoparticles. Theranostics 2013; 3(4):282-288. 


\section{Author Biography}

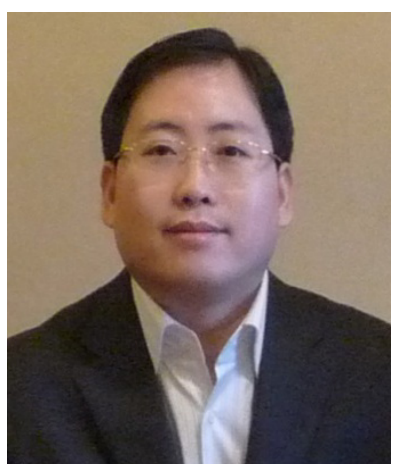

Guanying Chen received his B.S. and Ph.D. degree in optics in 2004, and 2009, respectively, from Harbin Institute of Technology, P. R. China. He then worked as a postdoctoral fellow (2010-2011) with Professor Paras N. Prasad at the Institute for Lasers, Photonics and Biophotonics, University at Buffalo, State University of New York, and Professor Hans Ågren at Department of Theoretical Chemistry \& Biology, Royal Institute of Technology. He is a research assistant professor at the Institute for Lasers, Photonics, and Biophotonics, at University at Buffalo, State University of New York. He holds a joint affiliation with Harbin Institute of Technology. His interests include photon up-conversion and down-conversion nanomaterials, nanostructured solar cells, and nanoparticles-based diagnostics and therapy.

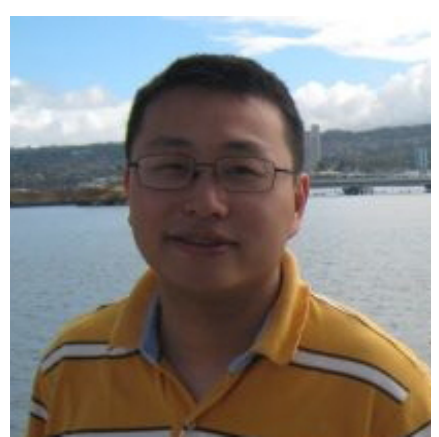

Gang Han received his B.Sc. and M.S. degrees in Chemistry from Nanjing University, and his Ph.D. degree in Chemistry from University of Massachusetts-Amherst. He was a postdoctoral scholar at the Molecular Foundry, Lawrence Berkeley National Lab. He is currently an Assistant Professor in the Biochemistry and Molecular Pharmacology Department at University of Massachusetts- Medical School. His current research focuses on the development of biocompatible synthetic nanomaterials for bioimaging and therapy. 